\title{
A primeira tese brasileira sobre a alienação mental: leituras, plágios e ciência
}

\author{
Ana Maria Galdini Raimundo Oda
}

Este artigo apresenta o texto "Considerações gerais sobre a alienação mental" (Peixoto, 1837), considerada a primeira tese na área de psiquiatria no Brasil. Seu autor copiou literalmente extensos trechos de dois dicionários médicos franceses, o Dictionnaire de médecine et de chirurgie pratiques $e$ o Dictionnaire des sciences médicales. Não se trata de apropriação destes textos científicos, mas de plágio, na maior parte da tese. O especifico contexto de produção desta e de outras teses de alunos das Faculdades de Medicina do século XIX deve ser considerado, quando se analisa a história da psiquiatria no país, relativizando o papel das teses como expressão local do alienismo, ou como indicativas de uma produção acadêmica típica das Faculdades de Medicina.

Palavras-chave: Antonio Luiz da Silva Peixoto, teses de medicina - século XIX, dicionários médicos - século XIX, alienismo 
A seção "História da Psiquiatria" da Revista Latinoamericana de Psicopatologia Fundamental republica a tese Considerações gerais sobre a alienação mental, de Antonio Luiz da Silva Peixoto, aluno da Faculdade de Medicina do Rio de Janeiro. A tese foi defendida em 1837, e é considerada a primeira a tratar deste tema (Moreira, 1908; Machado e cols., 1978; Engel, 2001), sendo citação recorrente nos estudos sobre a psiquiatria no país.

\section{Sobre as teses de medicina}

Durante o período regencial, uma reforma do ensino médico transformou as duas Academias Médico-Cirúrgicas existentes em Faculdades de Medicina da Bahia e do Rio de Janeiro. As Academias concediam apenas os títulos de "cirurgião formado" ou de "cirurgião aprovado", e as Faculdades, a partir de 1832, passaram a dar o título de doutor em medicina, obtido pelo aluno que concluísse os seis anos de estudos e defendesse, diante da Congregação de lentes, uma tese escrita em português ou em latim, impressa à sua custa (Santos Filho, 2002).

Para a feitura das teses, uma lista de temas ou "pontos" era determinada a cada ano pelas Faculdades, referentes às diversas cadeiras ou seções de ensino médico, e um tema era escolhido pelo aluno ou lhe era atribuído por sorteio (o que varia conforme o período). A estrutura da tese, em geral, era composta por três partes: a primeira e principal tinha um tema desenvolvido como ensaio ou monografia, a "dissertação"; a segunda, dedicava-se a "proposições" sobre assuntos relacionados às seções do curso, que eram escritas em forma de sentenças breves, numeradas; "aforismos hipocráticos" em latim, muito raramente em grego, encerravam a tese. As primeiras teses, das décadas de 1830/1850, muitas vezes se resumem a quatro ou cinco páginas, e tendem a aumentar de volume no decorrer do tempo. A elaboração das teses parece refletir as diferenças individuais entre os alunos que concluíam o curso de medicina: alguns parecem estar interessados apenas em cumprir a formalidade burocrática para receber o título. Com relação à área da medicina mental, entre as décadas de 1860 e 1880, nas duas Faculdades, 
os temas se tornam cada vez mais específicos, tais como sintomas psiquiátricos e entidades nosológicas, suicídio, aspectos legais da alienação mental, tratamentos das enfermidades mentais e o papel da civilização e do progresso em seu desenvolvimento. Observe-se que a psiquiatria não era disciplina autônoma até a década de 1880, no ensino médico brasileiro; assim, os temas psicológicos ou psicopatológicos se distribuíam nas disciplinas de três grandes áreas: seção clínica ou de ciências médicas, seção cirúrgica e seção de ciências acessórias. ${ }^{1,2}$

\section{A tese de Peixoto e o discurso sobre a necessidade do hospício de alienados}

Quanto ao autor de Considerações gerais sobre a alienação mental, quase nada sabemos, além de que era natural do Rio de Janeiro e que não tem outros trabalhos publicados conhecidos. Depois da formatura, vamos encontrá-lo atendendo em sua residência à Rua Nova de São Bento, onde se prestava a "todos os misteres de sua profissão" e também oferecia consultas gratuitamente aos indigentes, "todos os dias em sua casa de manhã, das 7 às 8 horas, no verão, e das 8 às 9, no inverno", conforme anúncio veiculado no Diário do Rio de Janeiro, em 1838.

A tese é um trabalho essencialmente teórico, no decorrer do qual Peixoto faz menções ocasionais a casos de loucura vistos ou sabidos por ele e também alguns comentários sobre a situação dos alienados no Rio de Janeiro e sobre o tratamento destes no Hospital da Santa Casa de Misericórdia. ${ }^{3}$ Neste ponto, ele acusa a Santa Casa de manter os loucos em local sem "regímen, limpeza, polícia e caridade",

${ }^{1}$ Os comentários sobre as teses são resultados parciais referentes a pesquisa financiada pela Fundação de Amparo à Pesquisa do Estado de São Paulo. ODA, Ana Maria G. R. Relatório científico final do projeto "Dos desgostos provenientes do cativeiro: uma história da psicopatologia dos escravos brasileiros no século XIX”. Fapesp processos 04/00442-4 (Auxílio-Pesquisa) e 04/07810-9 (Bolsa). Campinas, agosto de 2008, p. 170-181.

${ }^{2}$ A criação da cadeira de Psiquiatria nas duas Faculdades de Medicina data de outubro de 1882 (Moreira, 1908), decorrente da reforma do ensino médico iniciada em 1879, que instituiu várias novas cadeiras, tendo sido realizados os primeiros concursos para a cátedra, e para os respectivos adjuntos e auxiliares de ensino prático, em 1883 (Nascimento, 1908).

Para uma visão geral sobre a organização do ensino médico nas duas Faculdades, consultar o trabalho de Santos Filho (2002); sobre a Faculdade do Rio de Janeiro, ver Ferreira, Fonseca e Edler (2001).

${ }^{3}$ A Faculdade de Medicina do Rio de Janeiro funcionou na Santa Casa de Misericórdia até 1836, e as aulas práticas e observações clínicas eram feitas em suas enfermarias (Santos Filho, 2002). 
defendendo a necessidade de hospícios especiais para alienados, repetindo uma crítica que já vinha sendo feita pelos médicos desde o início da década de 1830 . Em 1831, o relatório da Comissão de Salubridade da Sociedade de Medicina do Rio de Janeiro (SMRJ), presidida por José Martins da Cruz Jobim, também professor de Medicina Legal da Faculdade, considerava as instalações do hospital insalubres e o tratamento reservado aos loucos como "o cúmulo da barbaridade" (Jobim e cols., 1831, p. 79). Depois, um artigo de José Francisco Xavier Sigaud, um dos fundadores da SMRJ, igualmente criticava a Santa Casa, assinalando que o tratamento dos alienados deveria sair do âmbito da caridade e entrar para o da filantropia, entendida como ação do Estado monárquico voltada para os pobres, dirigida pelos preceitos da higiene pública, e em estabelecimento especial (Sigaud, 1835). ${ }^{4}$

Ao final da tese, o autor refere os tratamentos físicos e o tratamento moral seguidos pelo médico assistente do Hospital da Santa Casa, e que lhes foram relatados pelo próprio Luiz Vicente De-Simoni, médico e literato italiano, outro dos fundadores da SMRJ. Faz uma crítica direta ao que seria o uso indevido do "tronco" (instrumento que ali estava para punição dos escravos faltosos) para amarrar os loucos furiosos, como meio moral de repressão, o que considera "um ato de degradação", uma vez que se trata de pessoas livres. Diz Peixoto supor "que é talvez a lei da necessidade que leva o Sr. Dr. de Simoni a usar de semelhante meio (...)". Esse "talvez" ofenderia o médico italiano a ponto de ser retomado e comentado em artigo publicado dois anos depois, na Revista Médica Fluminense, um longo e eloquente trabalho intitulado "Importância e necessidade da criação de um manicômio ou estabelecimento especial para o tratamento dos alienados" (De-Simoni, 1839), cujo título é autoexplicativo.

\section{Um estudante, dois dicionários: como fazer uma tese}

$\mathrm{Na}$ tese, o autor trata das causas, sintomas, marcha, duração, terminação, diagnóstico, prognóstico, anatomia patológica, sede, natureza e tratamento da alienação

${ }^{4}$ Como se sabe, tendo a construção iniciada em 1841, o primeiro hospício do país, o Hospício de Pedro II, modelar e emblema de modernidade, iniciaria seu funcionamento em 1852. No decorrer do Segundo Reinado, foram criados hospícios para alienados em várias províncias, sendo todos asilos muito precários (Oda e Dalgalarrondo, 2004). Somente perto da proclamação da República os alienistas efetivamente assumiram a direção do então denominado Hospício Nacional, bem como dos diversos hospícios de alienados brasileiros, deslocando as administrações leigas das Santas Casas e as ordens religiosas que ali serviam, em processo que se consolidou em começos do século XX (sobre este processo, ver Machado e cols., 1978; Engel, 2001; Teixeira e Ramos, 2012). 
mental. Como o leitor tem à sua disposição o texto integral, apenas indicaremos a seguir os pontos relevantes dele; ou melhor, o modus operandi do autor para produzi-lo.

Conforme observado pelos pesquisadores que se debruçaram sobre as teses de medicina, estas eram exercícios teóricos acadêmicos, nem sempre originais (Santos Filho, 2002; Machado e cols., 1978). A escolha dos temas não implicava escolha de uma especialidade e, no caso da alienação mental ou de temas correlatos, tampouco se correlacionava com a prática alienista. Nessa época, as teses seriam a reprodução de ideias ditas francesas, sobretudo de Esquirol, tal como na tese de Peixoto, de acordo com Machado e cols. (1978). Ressalta Magali Engel que Antonio Luiz Peixoto descreve poucos aspectos concretos da loucura no Rio de Janeiro (diferentemente dos citados Sigaud e De-Simoni) e que, quando defende o isolamento dos loucos, e indica as características da alienação e o seu tratamento, o faz baseado em referências teóricas, elencadas no correr do seu texto (Pinel, Esquirol, Frank, Foville, Georget, Dubois, Broussais etc.). Segundo Engel, essas referências poderiam indicar um aprofundamento teórico do autor da tese no campo do alienismo, quiçá adquirido nos estudos feitos durante a sua elaboração (2001, p. 199).

Também acreditávamos na erudição do doutorando Peixoto, ou no seu esforço em consultar e sintetizar os vários autores referidos na sua tese, até que acessamos a fonte assim indicada no texto original: "Foville, no seu artigo sobre a alienação mental, no Dicc. de Med. e Cir. Prat. (...)". Trata-se do conhecido Dictionnaire de médecine et de chirurgie pratiques, uma enciclopédia médica editada por Gabriel Andral e colaboradores em 15 volumes, entre 1829 e 1836, e cujo verbete assinado por A. Foville, aliénation mentale, encontra-se no tomo I (1829, p. 484-586). Efetivamente, nesse verbete encontramos o trecho mencionado na tese, e muito mais. À medida que comparávamos o verbete e a tese, notamos que Peixoto usou um expediente similar ao que mais tarde usaria Joaquim Manoel de Macedo, em sua tese Considerações sobre a nostalgia (1844). Como indicamos em outro trabalho, Macedo referiu ali certos autores como se os houvera lido diretamente, mas realmente recorreu à cópia de vários trechos de dicionários médicos, por exemplo, do Dictionnaire des sciences médicales editado por Panckoucke (Oda, 2008, p. 751). ${ }^{5}$

${ }^{5}$ A tese de Macedo tem várias referências bibliográficas (médicas e literárias) em notas rodapé, mas muitas delas foram visivelmente retiradas de dicionários médicos, não de leitura direta das obras citadas. Por exemplo, ele faz uma citação sobre o suicídio de escravos nas colônias francesas (1844, p. 19), que foi integralmente copiada do verbete nostalgie do Dictionnaire des sciences médicales editado por Panckoucke (1819, p. 269), sem mencionar este dicionário na tese (Oda, 2008). 
Constatamos que a maior parte da tese de Peixoto repete literalmente trechos inteiros do extenso verbete aliénation mentale do Dictionnaire de médecine et de chirurgie pratiques, traduzidos sem menção da fonte, ainda que cite o nome de Foville muitas vezes. Outra parte substancial da tese é cópia inconfessa de mais um longo verbete enciclopédico. Foville incluiu entre as referências do seu verbete aliénation os artigos folie, manie, monomanie, démence e idiotisme, vindos do Dictionnaire des sciences médicales, publicado pela editora Panckoucke entre 1812 e 1822, em 60 volumes. ${ }^{6}$ A segunda fonte extensamente copiada por Peixoto é o verbete folie, deste Dictionnaire des sciences médicales, assinado por Esquirol (1816, p. 151-240), dicionário cujo nome não é citado na tese.

Ao menos o primeiro dos dicionários referidos devia existir na Biblioteca da Faculdade de Medicina do Rio de Janeiro, onde o autor poderia tê-lo consultado. Em 1834, uma comissão formada pelos professores Manoel de Valladão Pimentel, Francisco Júlio Xavier e José Martins da Cruz Jobim, listara os livros que deveriam ser adquiridos para a sua Biblioteca (Ferreira, Fonseca, Edler, 2001, p. 69). Francisco Lobo reproduziu tal lista de obras encomendadas pela Faculdade, ${ }^{7}$ e ali encontramos nomeado o Dictionnaire de médecine et de chirurgie pratiques (Lobo, 1982, p. 25).

É importante observar que aqui não se trata de apropriação teórica desses dois textos científicos, ou de ressignificação dos textos lidos, mas de cópia literal e extensa, igualmente de informações relevantes ou de frases de efeito, com algum adendo pessoal, raramente com sínteses do lido. Em resumo, trata-se de plágio, em parte substantiva da tese de Peixoto. No caso de Joaquim Manoel de Macedo, mais complexo que o de Peixoto, existe uma transformação de sentido dos textos lidos e copiados, um arranjo próprio em certa direção, o que faria dele também um autor, não somente um plagiador. Com seu texto, aquele jovem médico-romancista, identificado à classe senhorial, está interessado em defender, além da tese acadêmica, a abolição do tráfico negreiro e a manutenção da escravidão, e usa argumentos de diferentes fontes para tal (Oda, 2008).

${ }^{6}$ Tais verbetes seriam reunidos posteriormente por Esquirol em seu tratado Des maladies mentales: considérées sous les rapports médical, hygiénique et médico-légal.

${ }^{7}$ A lista foi remetida em ofício ao governo regencial, em julho de 1834, para que as obras fossem compradas em Paris. Porém, apenas parte das obras foi adquirida, com recursos da própria Faculdade. As obras pedidas estão enumeradas segundo as áreas - Química Médica e Mineralogia; Física Médica; Botânica e Zoologia; Anatomia; Fisiologia; Partos e Moléstias de Crianças; Higiene e História da Medicina; Farmácia e Matéria Médica; Patologia e Clínica Externa; Patologia e Clínica Interna; Medicina Legal; Jornais e Obras Diversas. Em 1839, a Biblioteca tinha 1392 volumes, referentes a 489 obras, obtidas por meio de doações e aquisições (Lobo, 1982). 
Vale lembrar que o conceito de propriedade literária estava definido desde o século XVIII, a partir da identidade da obra reconhecível em suas várias formas de publicação (Chartier, 2012). Vigia então nas ciências a prática de enumerar a bibliografia: tanto o Dictionnaire des sciences médicales quanto o Dictionnaire de médecine têm listas das obras usadas em sua confecção, ao final de cada verbete. As listas de referência dos artigos enciclopédicos de Foville e de Esquirol mostram que o chamado alienismo "francês" bebia de variadas fontes (latinas, alemãs, inglesas, norte-americanas, além das francesas). Assim, o que circula no dicionário sob a autoria de Esquirol representa a síntese e a apropriação (na acepção de produção de novos sentidos) de muitas fontes, e da mesma forma no caso de Foville. Enciclopédias e dicionários médicos veiculavam a consolidação do saber sancionado, neste sentido contribuindo na construção de obras autorais (Chartier, 2012) no campo psiquiátrico.

Apenas como ilustração, indicamos a localização de alguns trechos, relacionando as seções da tese de Peixoto às páginas dos dicionários de onde foram retirados parágrafos copiados:

Dictionnaire de médecine et de chirurgie pratiques (tomo I, 1829):

Sintomatologia: p. 486-503; p. 510-513.

Invasão, marcha: p. 523-531.

Diagnóstico: p. 559-560.

Prognóstico: p. 560-561.

Anatomia Patológica: p. 531-559.

Sede e natureza: p. 531-532.

Dictionnaire des sciences médicales (volume 16, 1816):

Etiologia: p. 164-195.

Tratamento: p. 218-223; p. 230-237.

\section{Concluindo}

Atualmente, sítios eletrônicos abrigam coleções de impressos digitalizados e oferecem acesso integral a obras relevantes dos séculos passados, entre as quais muitos dicionários médicos, recursos valiosos onde se pode investigar a construção e a circulação de conhecimentos científicos. E, porque não dizer, fornecem recursos que facilitam a detecção de alguns plágios mais ou menos impudentes de acadêmicos premidos pelo tempo e pela obrigação de publicar sua tese de medicina.

Como muitos colegas seus, Antonio Luiz da Silva Peixoto afirma que escrevia a tese para satisfazer uma exigência legal, e se desculpa por sua insuficiência acadêmica, o que neste caso parece não ter sido apenas um artifício retórico. A despeito 
dos plágios que identificamos ao cotejar a tese e os dois dicionários médicos franceses, a monografia de Peixoto segue tendo seu valor como documento histórico. Faz refletir sobre a relativização do papel atribuído às teses de medicina, em especial às das primeiras décadas de produção, como expressão local do alienismo, e mesmo como referidas a um projeto de intervenção social da medicina, no sentido de remover os loucos de circulação. E ainda, questionar o quanto elas podem ser consideradas expressão de uma produção acadêmica "típica" das Faculdades de Medicina.

Mais adiante, a maneira como se produziam as teses de medicina em Paris, na década de 1880, seria apresentada como problemática pelo professor J. L. Petit, em forma de crítica irônica, que se iniciava assim: "Há muitos meios de fazer uma tese. Certamente o mais cômodo é encarregar alguém deste trabalho" (1891, p. 374). O professor da Faculdade de Medicina da Bahia, Raimundo Nina-Rodrigues, ponderava que as críticas de Petit eram muito pertinentes e aplicáveis às teses das nossas Faculdades de Medicina (1891, p. 372), tanto que publicou o texto em português, na Gazeta Medica da Bahia, recomendando aos professores que o lessem.

\section{Referências}

Aliénation Mentale (A. Foville). In Andral, G.; Bégin, É.-A.-N.-J.; Blandin, P. F. (eds.). (1829). Dictionnaire de médecine et de chirurgie pratiques. Tome I (A-ALIEN). Paris: Gabon, Méquignon-Marvis, J.-B. Baillière, Crochard, p. 484-586. Recuperado em 18 de novembro de 2013, de: <http://www.biusante.parisdescartes.fr/histmed/medica/cote?34826x01>

Chartier, R. (2012). O que é um autor: revisão de uma genealogia. ( Luzmara Curcino e Cláudio Eduardo Bezerra, Trad.). São Carlos: Edufscar.

De-Simoni, L. V. (1839, set.). Importância e necessidade da criação de um manicômio ou estabelecimento especial para o tratamento dos alienados. Revista Médica Fluminense, V(6), 241-62. [Republicado em Revista Latinoamericana de Psicopatologia Fundamental, 7(1), 142-59, 2004].

Diário do Rio de Janeiro (1838). Anúncios particulares. Edição 54. Rio de Janeiro: Thypographia do Diário, 1838. Recuperado em 18 de novembro de 2013, de: <http://hemerotecadigital. bn.br>

Engel, M. G. (2001). Delírios da razão: médicos, loucos e hospícios. Rio de Janeiro 1830-1930. (pp. 183-252). Rio de Janeiro: Fiocruz, 2001.

Ferreira, L. O.; Fonseca, M. R. F. da; Edler, F. C. (2001). A Faculdade de Medicina do Rio de Janeiro no século XIX: a organização institucional e os modelos de ensino. In Dantes, M. A. M. (Org.). Espaços da ciência no Brasil (1800-1930). (pp. 59-80). Rio de Janeiro: Fiocruz.

Folie (Esquirol). In Une Societé de Médecins et de Chirurgiens (ed.) (1916). Dictionnaire des sciences médicales. Volume 16 (FIS-FRA). Paris: Panckoucke, 1816, p. 151-240. Recu- 
perado em 18 de novembro de 2013, de: http://www.biusante.parisdescartes.fr/histmed/ medica/cote?47661x16>

Jobim, J. M. da C.; Silva, J. J.; Santos, C. J. (1831, abr.). Relatório da Comissão de Salubridade Geral, da Sociedade de Medicina do Rio de Janeiro, apresentado e aprovado na sessão de 19 de junho (de 1830). Semanário de Saúde Pública, 15, 77-81.

Lobo, F. B. (1982, jan./mar.). A Biblioteca da Faculdade de Medicina do Rio de Janeiro no Império. Revista do Instituto Histórico e Geográfico Brasileiro, Rio de Janeiro, 334, 19-37.

Macedo, J. M. de M. (1844). Considerações sobre a nostalgia. Tese apresentada à Faculdade de Medicina do Rio de Janeiro em 11 de dezembro de 1844. Rio de Janeiro: Typographia Imparcial de Francisco de Paula Brito.

Machado, R.; Loureiro, A.; Luz, R.; Muricy, K. (1978). Danação da norma: medicina social e constituição da psiquiatria no Brasil. (pp. 375-492). Rio de Janeiro: Graal.

Moreira, J. (1908). Psiquiatria. In Academia Nacional de Medicina (ed.). Em comemoração ao centenário do ensino médico. (pp. 432-448). Rio de Janeiro: Tipografia do Jornal do Commercio.

Nascimento, A. (1908). Organização do ensino médico. In Academia Nacional de Medicina (ed.). Em comemoração ao centenário do ensino médico. (pp. 47-55). Rio de Janeiro: Tipografia do Jornal do Commercio.

Nina-Rodrigues, R. (1891). Introdução ao artigo de J. L. Petit (Como se faz uma tese. Como de devia fazer). Gazeta Medica da Bahia, Salvador, 22, 372-373.

Oda, A. M. G. R.; Dalgalarrondo, P. (2004, mar.). O início da assistência aos alienados no Brasil ou importância e necessidade de estudar a história da psiquiatria. Revista Latinoamericana de Psicopatologia Fundamental, São Paulo, 7(1), 128-41.

Oda, A. M. G. R. (2008, dez.). Escravidão e nostalgia no Brasil: o banzo. Revista Latinoamericana de Psicopatologia Fundamental, São Paulo, 11(4), 735-761, (Suplemento).

Petit, J. L. (1891). Como se faz uma tese. Como se devia fazer. Gazeta Medica da Bahia, Salvador, 22, 372-373.

Santos Filho, L. (2002). Medicina no Período Imperial. In Holanda, S. B. de (Org.). O Brasil monárquico. (História geral da civilização brasileira, tomo II, vol. 3, pp. 467-471). Rio de Janeiro: Bertrand Brasil.

Sigaud, J. F. X. (1835, abr.). Reflexões acerca do trânsito livre dos doidos pelas ruas da cidade do Rio de Janeiro. Diário de Saúde ou efemérides das ciências médicas e naturais do Brasil, I(1), 6-8. [Republicado em Revista Latinoamericana de Psicopatologia Fundamental, São Paulo, 8(3), 559-62, set. 2005].

Teixeira, M. O. L.; Ramos, F. A. de C. (2012, jun.). As origens do alienismo no Brasil: dois artigos pioneiros sobre o Hospício de Pedro II. Revista Latinoamericana de Psicopatologia Fundamental, São Paulo, 15(2), 364-381. 


\section{Resumos}

(The first Brazilian thesis on mental alienation: readings, plagiarism and science)

This article presents the text entitled (English translation) "General Considerations on Mental Alienation" (Peixoto, 1837), considered the first thesis in the field of psychiatry in Brazil. Its author literally copied long excerpts from two French medical dictionaries, the Dictionnaire de médecine et de chirurgie pratiques and Dictionnaire des sciences médicales. This was not simple scientific appropriation of texts, but plagiarism, comprising almost the entire thesis. The specific context of the production of this and other theses of students at the faculties of medicine in the nineteenth century should be considered when analyzing the history of Brazilian psychiatry. This approach questions the role of theses as local expressions of alienism or as indicative of typical academic production at faculties of medicine.

Key words: Antonio Luiz da Silva Peixoto, medical theses - nineteenth century, medical dictionaries - nineteenth century, alienism

(La première thèse brésilienne sur l'aliénation mentale: lectures, plagiat et science)

Cet article présente le texte Considérations Générales sur l'Aliénation Mentale (Peixoto, 1837), considéré comme la première thèse dans le domaine de la psychiatrie au Brésil. Son auteur a littéralement copié de grands extraits de deux dictionnaires médicaux français, le Dictionnaire de Médecine et de Chirurgie Pratiques et le Dictionnaire des Sciences Médicales. Il ne s'agit pas d'appropriation scientifique de ces textes, mais de plagiat, constituant la plus grande partie de la thèse. Le contexte spécifique de la production de cette et d'autres thèses rédigées par les étudiants des facultés de médecine du XIXe siècle doit être pris en compte lors de l'analyse de l'histoire de la psychiatrie au Brésil, relativisant le rôle des thèses comme une expression locale de l'aliénisme, soit comme indice d'une production universitaire typique des facultés de médecine.

Mots clés: Antonio Luiz da Silva Peixoto, thèses médicales - XIX ${ }^{\mathrm{e}}$ siècle, dictionnaires médicaux - XIX ${ }^{\mathrm{e}}$ siècle, aliénisme

(La primera tesis brasileñna sobre la alienación mental: lecturas, plagios y ciencia)

Este artículo presenta el texto Consideraciones generales sobre la alienación mental (peixoto, 1837), considerado la primera tesis en el área de la psiquiatria en Brasil. Su autor copió literalmente trechos largos de dos diccionarios médicos franceses, el Dictionnaire de médecine et de chirurgie pratiques e el Dictionnaire des sciences médicales. No fue una apropiación de estes textos cientificos, sino de plagio en la mayor parte de la tesis. El contexto especifico de esta y de otras tesis de alumnos de las Facultades de Medicina del siglo XIX debe ser considerado cuando se analisa la historia de la psiquiatria en el país. Relativizando el papel de las tesis como expresión local del 
alienismo, o como indicativas de una producción académica típica de las Facultades de Medicina.

Palabras clave: Antonio Luiz da Silva Peixoto, tesis de medicina - siglo XIX, diccionarios médicos - siglo XIX, alienismo

(Die erste brasilianische These über geistige Verwirrung: Lektüre, Plagiate und Wissenschaft)

In diesem Beitrag wird der Text Considerações gerais sobre a alienação mental (Peixoto, 1837), (Allgemeine Überlegungen zum Thema geistige Verwirrung) besprochen. Er gilt als erste These im Bereich der Psychiatrie in Brasilien. Der Autor hat regelrecht lange Abschnitte aus zwei französischen medizinischen Wörterbüchern, dem Dictionnaire de médecine et de chirurgie pratiques und dem Dictionnaire des sciences médicales abgeschrieben. Dies gilt jedoch nicht als Aneignung dieser wissenschaftlichen Texte, sondern ist Plagiat, und zwar zum größten Teil der These. Es ist wichtig, den spezifischen Produktionskontext dieser und anderer wissenschaftlicher Abhandlungen der Medizinstudenten des 19.Jahrhunderts zu beachten, wenn man die Geschichte der Psychiatrie in Brasilien untersucht. Die Rolle der Thesen muss relativiert werden und als lokale Äußerung zum Thema Wahnsinn betrachtet werden, oder als Hinweise auf typische akademische Abhandlung der Medizinfakultäten.

Schlüsselwörter: Antonio Luiz da Silva Peixoto; medizinische Thesen - 19. Jahrhundert; medizinische Wörterbücher - 19. Jahrhundert; Verwirrung Entfremdung

Citação/Citation: Oda, A. M. G. R. (2013, dezembro). A primeira tese brasileira sobre a alienação mental: leituras, plágios e ciência. Revista Latinoamericana de Psicopatologia Fundamental, 16(4), 630-641.

Editor do artigo/Editor: Prof. Dr. Paulo Dalgalarrondo e Profa. Dra. Ana Maria Galdini Raimundo Oda

Recebido/Received: 10.11.2013/ 11.10.2013 Aceito/Accepted: 20.11.2013 / 11.20.2013

Copyright: (C) 2009 Associação Universitária de Pesquisa em Psicopatologia Fundamental/ University Association for Research in Fundamental Psychopathology. Este é um artigo de livre acesso, que permite uso irrestrito, distribuição e reprodução em qualquer meio, desde que o autor e a fonte sejam citados / This is an open-access article, which permits unrestricted use, distribution, and reproduction in any medium, provided the original author and source are credited. 
Financiamento/Funding: Esta pesquisa é financiada pela Fundação de Amparo à Pesquisa do Estado de São Paulo - Fapesp (São Paulo, SP, Br) / This research is funded by Fundação de Amparo à Pesquisa do Estado de São Paulo - Fapesp (São Paulo, SP, Br).

Conflito de interesses/Conflict of interest: $\mathrm{O}$ autor declara que não há conflito de interesses / The author declares that has no conflict of interest.

\section{Ana Maria Galdini Raimundo Oda}

Médica psiquiatra, com mestrado em Saúde Mental (1998) e doutorado em Ciências Médicas (2003) pela Universidade Estadual de Campinas - Unicamp (Campinas, SP, Br). Editora associada da Revista Latinoamericana de Psicopatologia Fundamental (seção História da Psiquiatria); Atualmente é professora adjunta do Departamento de Medicina do Centro de Ciências Biológicas e da Saúde da Universidade Federal de São Carlos - Ufscar (São Carlos, $\mathrm{SP}, \mathrm{Br}$ ).

Caminho 03, n. 104B, Cond. Bosques de Notre Dame, Distrito de Sousas 13104-902 Campinas, SP, Br anamariagr.oda@gmail.com 